\title{
Semi-stable fibrations of generic $p$-rank 0
}

\author{
Junmyeong Jang \\ email : jang3@math.purdue.edu \\ Mathematics Subject Classification : 11G25, 14J20
}

\section{Introduction}

Let $k$ be an algebraically closed field and $\pi: X \rightarrow C$ be a semi-stable fibration of a connected proper smooth surface to a connected proper smooth curve over $k$. If the base field $k$ is a subfield of $\mathbb{C}$, the filed of complex numbers, the following semi-positivity theorem holds.

Theorem.(Semi-Positivity Theorem, Xiao) If $\pi: X \rightarrow C$ is a fibration of a proper smooth surface to a proper smooth curve over $\mathbb{C}$, then all the quotient bundles of $\pi_{*} \omega_{X / C}$ are of non-negative degree. [10],p.1

In general the semi-positivity theorem is not valid over a field of positive characteristic. In [7], Moret-Bailly constructed a semi-stable fibration $\pi_{M}: X_{M} \rightarrow \mathbb{P}^{1}$ of fiber genus 2 such that $R^{1} \pi_{M *} \mathcal{O}_{X_{M}}=\mathcal{O}(1) \oplus \mathcal{O}(-p)$ where $p$ is the characteristic of the base filed. In the previous work, [6] we have proved that for a semi-stable fibration $\pi: X \rightarrow C$, if the generic fiber is ordinary, then the semi-positivity theorem holds. Precisely, when the generic fiber of $\pi$ is ordinary, all the Harder-Narasimhan slopes of $R^{1} \pi_{*} \mathcal{O}_{X}$ are nonpositive. For Moret-Bailly's example, the $p$-rank of the generic fiber is 0 . In particular, every special fiber of $\pi_{M}$ is a supersingular smooth curve of genus 2 or a union of two supersingular elliptic curves which intersect at a point transversally. In this paper we prove the following theorem which generalizes the failure of the semi-positivity theorem for Moret-Bailly's example.

Theorem 1. Let $\pi: X \rightarrow C$ be a non-isotrivial semi-stable fibration of proper smooth surface to a proper smooth curve over a field of positive characteristic. If the generic $p$-rank of $\pi$ is 0 , then $F_{C}^{n *} R^{1} \pi_{*} \mathcal{O}_{X}$ has a positive Harder-Narasimhan slope for a sufficiently large $n \in \mathbb{N}$. In particular, if the genus of $C$ is 0 or $1, R^{1} \pi_{*} \mathcal{O}_{X}$ has a positive Harder-Narasimhan slope.

As an application of the theorem, we obtain a result on a distribution of $p$-ranks of reductions of a certain non-closed point in the moduli space of curves over a number field. 
Corollary 2.8. Suppose that $\pi: X \rightarrow C$ is a non-isotrivial semi-stable fibration of base genus 0 or 1 defined over a number field $F$, and that $U \subseteq C$ is the smooth locus of $\pi$. $\pi$ defines a non-constant morphism $f: U \rightarrow \mathcal{M}_{g, F}$. Let $P$ be the image of the generic point of $U$ under $f$. Then the reduction $P_{v}$ is not contained in the $p$-rank 0 strata for almost all $v$.

This result can be considered as a variation of Serre's ordinary reduction conjecture. It is a weak statement since it is only about some non-closed points in the moduli space and $0 p$-rank. But it is a somewhat interesting phenomenon that the semi-positivity theorem, which is concerned with a coherent module on a fiber of characteristic 0 , encodes an information of $p$-ranks of the reductions.

\section{Proof of Theorem 1.}

We follow the terminology of [6]. Let us recall the definition of a semi-stable curve. Let $k$ be an algebraically closed field and $C$ be a projective curve over $k$.

Definition 2.1. $C$ is (semi-)stable if

1. It is connected and reduced.

2. All the singular points are normal crossing.

3. An irreducible component, which is isomorphic to $\mathbb{P}^{1}$, meets other components in at least 3(resp. 2) points.

For an arbitrary base scheme, we define a (semi-)stable curve as follows.

Definition 2.2. A proper flat morphism of relative dimension 1 of schemes $\pi: X \rightarrow S$ is a (semi-)stable curve if every geometric fiber of $\pi$ is a (semi-)stable curve in the sense of definition 2.1.

In this paper, we assume $\pi: X \rightarrow C$ is a generically smooth semi-stable fibration of a proper smooth surface to a proper smooth curve over a field $k$ unless it is stated otherwise.

Definition 2.3. For a generically smooth semi-stable fibration $\pi: X \rightarrow C$ defined over a field of positive characteristic, the generic $p$-rank of $\pi$ is the $p$-rank of a geometric generic fiber of $\pi$.

\subsection{Self duality of $B \omega^{1}$}

Let $k$ be a perfect field of positive characteristic and $\pi: X \rightarrow C$ be a generically smooth semi-stable curve. Let $\omega_{X / C}^{1}$ be the relative dualizing line bundle for $\pi$. There is the canonical inclusion $i: \Omega_{X / C}^{1} \hookrightarrow \omega_{X / C}^{1}$. At a relative smooth point for $\pi, i$ is an 
isomorphic. On the other hand, at a relative singular point, where étale locally $\pi$ is given by

$$
\operatorname{Spec} k[x, y, t] /(x y-t) \rightarrow \operatorname{Spec} k[t],
$$

$\omega_{X / C}^{1}$ is a free module of rank 1 generated by $d x / x=-d y / y$ and $\Omega_{X / C}^{1}$ is a submodule of $\omega_{X / C}^{1}$ generated by $d x$ and $d y$ via $i$. Composing with the inclusion $i$, we have the differential morphism $d: \mathcal{O}_{X} \rightarrow \omega_{X / C}^{1}$. When $F_{X / C}$ is the relative Frobenius morphism for $\pi, \pi$

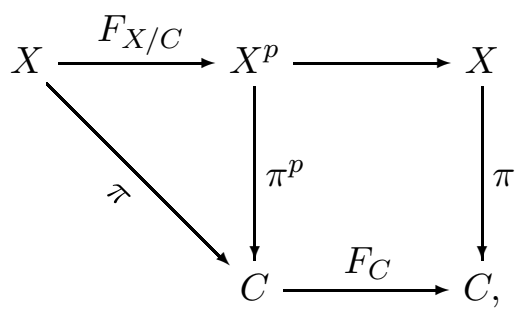

$d: F_{X / C *} \mathcal{O}_{X} \rightarrow F_{X / C *} \omega_{X / C}^{1}$ is $\mathcal{O}_{X p}$-linear. The kernel of $d$ is the image of $F_{X / C}^{*}$ : $\mathcal{O}_{X}{ }^{p} \hookrightarrow F_{X / C *} \mathcal{O}_{X}$ and the image of $d$ is denoted by $B^{1} \omega_{X / C}$ or $B^{1} \omega . B \omega^{1}$ is flat over $\mathcal{O}_{C}$. Let $U \hookrightarrow X$ be the smooth locus for $\pi$. The usual Cartier isomorphism

$$
C: \Omega_{U / C}^{1} / B^{1} \Omega_{U / C} \rightarrow \Omega_{U^{p} / C}
$$

is extended to an isomorphism

$$
C: \omega_{X / C}^{1} / B^{1} \omega \rightarrow \omega_{X^{p} / C}^{1} \cdot[5], p .381
$$

Using this Cartier morphism, we have an $\mathcal{O}_{X^{p}}$-linear paring

$$
F_{X / C *} \mathcal{O}_{X} \otimes F_{X / C *} \omega_{X / C}^{1} \rightarrow \omega_{X}^{1} / C,(\alpha, \omega) \mapsto C(\alpha \omega) .
$$

This pairing induces a pairing

$$
\left(F_{X / C *} \mathcal{O}_{X} / \mathcal{O}_{X^{p}}\right) \otimes B^{1} \omega \rightarrow \omega_{X}^{1} / C
$$

On $U$, this pairing gives a perfect self duality. In particular, we have

$$
B^{1} \Omega_{U / C} \simeq \underline{\operatorname{Hom}}\left(B^{1} \Omega_{U / C}, \Omega_{U^{p} / C}^{1}\right) .
$$

Proposition 2.4. If $X$ is a smooth surface over a perfect field $k$ which admits a semi-stable fibration, $\pi: X \rightarrow C$, to a smooth curve $C$ over $k$, then

$$
B^{1} \omega_{X / C} \simeq \underline{\operatorname{Hom}}\left(B^{1} \omega_{X / C}, \omega_{X^{p} / C}^{1}\right) .
$$

Proof. It's enough to check that the paring is perfect at the relative singular points in $X$. Let $x \in X$ be a relative singular point. Étale locally, we may assume $X=\operatorname{Spec} A$, $C=\operatorname{Spec} B$ where

$$
A=k[x, y, t] /(x y-t) \simeq k[x, y], B=k[t]
$$


and $\pi$ is the canonical morphism

$$
k[t] \rightarrow k[x, y, t] /(x y-t) .
$$

Let $A^{p}=A \otimes_{B}\left(B, F_{B}\right)$. Then $A^{p}=k[X, Y, t] /\left(X Y-t^{p}\right)$ and the relative Frobenius morphism is a $k$-algebra morphism $F_{A / B}: A^{p} \rightarrow A$ given by

$$
X \mapsto x^{p}, Y \mapsto y^{p} \text { and } t \mapsto x y .
$$

We may regard $A^{p}$ is a $k$-subalgebra of $A$ generated by $x^{p}, y^{p}, x y$. As an $A^{p}$-module, $B^{1} \omega=A / A^{p}$ is generated by

$$
x, x^{2}, \cdots, x^{p-1}, y, \cdots, y^{p-1} .
$$

$B^{1} \omega$ is a torsion free $A^{p}$-module and the $\operatorname{Frac}\left(A^{p}\right)$-dimension of $B^{1} \otimes_{A^{p}} \operatorname{Frac}\left(A^{p}\right)$ is $p-1$. Therefore there are only $p-1$ obvious relations

$$
t^{p-1} x=X y^{p-1}, t^{p-2} x^{2}=X y^{p-2}, \cdots, t x^{p-1}=X y
$$

among the generators and we have an $A^{p}$-module decomposition

$$
B^{1} \omega=\oplus_{i=1}^{p-1}<x^{i}, y^{p-i}>.
$$

On the other hand, $\omega_{A / B}^{1}$ is a rank 1 free $A$-module generated by $d x / x=-d y / y$ and $\omega_{A^{p} / B}^{1}$ is a rank 1 free $A^{p}$-module generated by $d X / X=-d Y / Y$. The Cartier morphism is the $A^{p}$-linear morphism satisfying

$$
\begin{array}{ccc}
d x / x & \mapsto & d X / X, \\
x d x / x & \mapsto & 0, \\
& \vdots & \\
x^{p-1} d x / x & \mapsto & 0, \\
y d x / x & \mapsto & 0, \\
& \vdots & \\
y^{p-1} d x / x & \mapsto & 0 .
\end{array}
$$

In the above decomposition of $B^{1} \omega$, it's easy to see that the dual of $\left\langle x^{i}, y^{p-i}\right\rangle$ is $<x^{p-i}, y^{i}>$ and that the pairing $B^{1} \omega \otimes B^{1} \omega \rightarrow \omega_{X^{p} / C}^{1}$ gives a perfect duality of the dual components of both sides. This proves the claim.

Corollary 2.5. Let $k$ be a perfect field of positive characteristic. Let $\pi: X \rightarrow C$ be a semi-stable fibration of a proper smooth surface to a proper smooth curve over $k$. Let $M$ and $T$ be the free part and the torsion part of $R^{1} \pi_{*} B^{1} \omega_{X / C}$ respectively and $N=R^{1} \pi_{*} \mathcal{O}_{X}$. Then there exists a exact sequence of coherent modules on $C$

$$
0 \rightarrow M^{*} \rightarrow F_{C}^{*} N \rightarrow N \rightarrow M \oplus T \rightarrow 0
$$


Proof. The exact sequence of coherent $\mathcal{O}_{X^{p}}$-modules

$$
0 \rightarrow \mathcal{O}_{X^{p}} \rightarrow F_{X / C *} \mathcal{O}_{X} \rightarrow B^{1} \omega_{X / C} \rightarrow 0
$$

gives a long exact sequence for the $\pi_{*}^{p}$ functor

$$
0 \rightarrow \mathcal{O}_{C} \cong \mathcal{O}_{C} \rightarrow \pi_{*}^{p} B^{1} \omega_{X / C} \rightarrow R^{1} \pi_{*}^{p} \mathcal{O}_{X} \rightarrow R^{1} \pi_{*}^{p} F_{X / C *} \mathcal{O}_{X} \rightarrow R^{1} \pi_{*}^{p} B^{1} \omega_{X / C} \rightarrow 0 .
$$

Because the Frobenius morphism of $C$ is finite flat, $R^{1} \pi_{*}^{p} \mathcal{O}_{X^{p}}=F_{C}^{*} R^{1} \mathcal{O}_{X}$. And the relative Frobenius morphism $F: X \rightarrow X^{p}$ is finite affine, so $R^{1} \pi_{*}^{p} F_{X / C *} \mathcal{O}_{X}=R^{1} \pi_{*} \mathcal{O}_{X}$. By proposition 2.4, $B^{1} \omega_{X / C} \simeq \underline{\operatorname{Hom}}\left(B^{1} \omega_{X / C}, \omega_{X^{p} / C}\right)$. Since $\pi$ is relative 1-dimensional, by the relative duality theorem

$$
\pi_{*}^{p} B^{1} \omega_{X / C}=\underline{\operatorname{Hom}}\left(R^{1} \pi_{*}^{p} B^{1} \omega_{X / C}, \mathcal{O}_{C}\right) .
$$

Therefore the claim follows.

\subsection{Proof of the theorem}

Theorem 1. Let $\pi: X \rightarrow C$ be a non-isotrivial semi-stable fibration of proper smooth surface to a proper smooth curve over a field of positive characteristic. If the generic $p$-rank of $\pi$ is 0 , then $F_{C}^{n *} R^{1} \pi_{*} \mathcal{O}_{X}$ has a positive Harder-Narasimhan slope for a sufficiently large $n \in \mathbb{N}$. In particular, if the genus of $C$ is 0 or $1, R^{1} \pi_{*} \mathcal{O}_{X}$ has a positive Harder-Narasimhan slope.

Proof. The $n$-iterative relative Frobenius morphism in the diagram

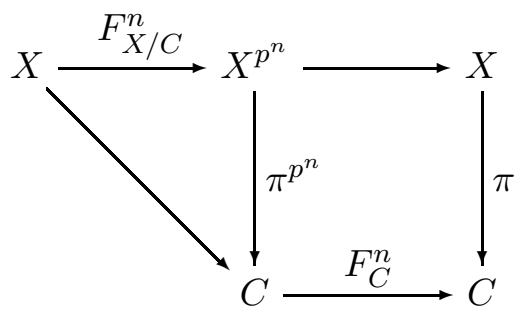

is the composition of relative Frobenius morphisms

$$
X \stackrel{F_{X / C}}{\rightarrow} X^{p} \stackrel{F_{X p / C}}{\rightarrow} \cdots \rightarrow X^{p^{n-1}} \stackrel{F_{X p^{n-1} / C}}{\longrightarrow} X^{p^{n}} .
$$

$F_{X / C}^{n}$ gives an exact sequence of coherent $\mathcal{O}_{X^{p^{n}}-\text { modules }}$

$$
(*) 0 \rightarrow \mathcal{O}_{X^{p^{n}}} \stackrel{F^{n *}}{\rightarrow} F_{*}^{n} \mathcal{O}_{X} \rightarrow E_{n} \rightarrow 0 .
$$

Here $E_{n}$ is flat over $\mathcal{O}_{C}$ and $E_{1}=B^{1} \omega_{X / C}$. If we denote $N=R^{1} \pi_{*} \mathcal{O}_{X}, R^{1} \pi_{*}^{p^{n}} \mathcal{O}_{X^{p^{n}}}=$ $F_{C}^{n *} N$. Let $\lambda_{n}: F_{C}^{n *} N \rightarrow N$ be the morphism induced by $F_{X / C}^{n *}$ in $(*)$. Because the relative Frobenius morphism commutes with a base change and the Frobenius morphism 
of $C$ is flat, $\lambda_{n}$ is the composition of Frobenius pullbacks of $\lambda_{1}: F_{C}^{*} N \rightarrow N$,

$$
\lambda_{n}=\lambda_{1} \circ \cdots \circ F_{C}^{(n-2) *} \lambda_{1} \circ F_{C}^{(n-1) *} \lambda_{1}: F_{C}^{n *} N \rightarrow F_{C}^{(n-1) *} N \rightarrow \cdots \rightarrow F_{C}^{*} N \rightarrow N .
$$

On the other hand, the restriction of $(*)$ to a special fiber $X_{s}$ is

$$
0 \rightarrow \mathcal{O}_{X_{s}^{p^{n}}} \rightarrow F_{X_{s} / k(s) *}^{n} \mathcal{O}_{X_{s}} \rightarrow E_{n, s} \rightarrow 0
$$

Furthermore we have the long exact sequence

$$
\cdots \rightarrow H^{1}\left(\mathcal{O}_{X_{s}^{p^{n}}}\right) \rightarrow H^{1}\left(\mathcal{O}_{X_{s}}\right) \rightarrow H^{1}\left(E_{n, s}\right) \rightarrow 0 .
$$

Since we have assumed that the generic $p$-rank of $\pi$ is 0 , by the Grothendieck specialization theorem, for all $s \in C$, the $p$-rank of $X_{s}$ is 0 . It follows that there exists $n \in \mathbb{N}$, such that $H^{1}\left(X_{s}, \mathcal{O}_{X_{s}^{p^{n}}}\right) \rightarrow H^{1}\left(X_{s}, \mathcal{O}_{X_{s}}\right)$ is the zero morphism for all $s \in C$ such that $X_{s}$ is smooth. Hence $\operatorname{dim} H^{0}\left(X_{s}, E_{n, s}\right)=\operatorname{dim} H^{1}\left(X_{s}, E_{n, s}\right)=\operatorname{gen} X_{s}=g$ for such $s$. But by the semi-continuity theorem, $\pi_{*}^{p^{n}} E_{n}$ and $R^{1} \pi_{*}^{p^{n}} E_{n}$ are vector bundles of rank $g$. Because $N$ is also a vector bundle of rank $g$ on $C$, considering the exact sequence

$$
0 \rightarrow \pi_{*}^{p^{n}} E_{n} \rightarrow F_{C}^{n *} N \stackrel{\lambda_{n}}{\rightarrow} N \rightarrow R^{1} \pi_{*}^{p^{n}} E_{n} \rightarrow 0,
$$

$\lambda_{n}=0$. Now assume all the Harder-Narasimhan slopes of $F_{C}^{i} N$ are non-positive for all $i$. Let $M$ be the free part of $R^{1} \pi_{*}^{p} B^{1} \omega_{X / C}$. Then $\pi_{*}^{p} B^{1} \omega_{X / C}=M^{*}=\underline{H o m_{\mathcal{O}_{C}}}\left(M, \mathcal{O}_{C}\right)$.(Corollary 2.4) All the Harder-Narasimhan slopes of $F_{C}^{i *} M^{*}$ are non-positive since $F_{C}^{i *} M^{*}$ is a subbundle of $F_{C}^{i *} N$, so all the Harder-Narasimhan slopes of $F_{C}^{i *} M$ are non-negative. Let us consider an exact sequence

$$
0 \rightarrow \operatorname{Im} \lambda_{i-1} / \operatorname{Im} \lambda_{i} \rightarrow N / \operatorname{Im} \lambda_{i} \rightarrow N / \operatorname{Im} \lambda_{i-1} \rightarrow 0 .(i \geq 2)
$$

We can also think of the free part of the above exact sequence

$$
0 \rightarrow V_{i}^{\prime} \rightarrow V_{i} \rightarrow V_{i}^{\prime \prime} \rightarrow 0
$$

Here $V_{i}$ is the free part of $N / \operatorname{Im} \lambda_{i}$ and $V_{i}^{\prime \prime}$ is the free part of $N / \operatorname{Im} \lambda_{i-1} \cdot V_{i}^{\prime}$ is the saturation of the free part of $\operatorname{Im} \lambda_{i-1} / \operatorname{Im} \lambda_{i}$ in $V_{i}$. We can see $V_{2}^{\prime \prime}=M$ is of non-negative degree by the assumption. On the other hand, since $V_{i}^{\prime}$ is a saturation of a quotient bundle of $F^{(i-1) *} M$, it is also of non-negative degree. Therefore, by induction $V_{i}$ is of non-negative degree. Since $\lambda_{n}=0$ for a sufficiently large $n$, the degree of $V_{n}=N$ is non-negative. But since $\pi$ is non-isotrivial, $\operatorname{deg} N$ is strictly negative. [9],p.173 It is contradiction, so $F^{i *} N$ has a positive Harder-Narasimhan slope for some $i$. If the genus of the base $C$ is 0 or 1 , the Frobenius pull back $F_{C}^{*}$ preserves the semi-stability of vector bundles, so $R^{1} \pi_{*} \mathcal{O}_{X}$ has a positive Harder-Narasimhan slope.

Remark 2.6. The failure of the semi-positivity theorem for Moret-Bailly's example in the introduction is a special case of Theorem 2. Using Theorem 2, we may construct a lot of counterexamples of the semi-positivity theorem. Assume $k$ is algebraically 
closed of positive characteristic. In $\mathcal{M}_{g, k}$, the moduli space of smooth proper curves of genus $g$ over $k$, the $p$-rank 0 strata is a closed subscheme which is purely $2 g-3$ dimensional.[3],p.120 Let $P$ be a 1 dimensional point in the $p$-rank 0 strata. By the semi-stable reduction theorem, [1],p.3 there is a semi-stable fibration $\pi: X \rightarrow C$ such that the morphism $C \rightarrow \mathcal{M}_{g, k}$ induced by $\pi$ sends the generic point of $C$ to $P$. Since the generic $p$-rank of $\pi$ is 0 , for a suitable Frobenius base change $\pi^{p^{n}}: X^{p^{n}} \rightarrow C$, $R^{1} \pi_{*}^{p^{n}} \mathcal{O}_{X^{p^{n}}}$ has a positive slope by Theorem 1. $X^{p^{n}}$ may contain isolated singularities. But the composition of $\pi^{p^{n}}$ and the desingularization $X^{(n)} \rightarrow X^{p^{n}}, \pi^{(n)}: X^{(n)} \rightarrow C$ is a semi-stable fibration and $R^{1} \pi_{*}^{(n)} \mathcal{O}_{X^{(n)}}=R^{1} \pi_{*}^{p^{n}} \mathcal{O}_{X^{p^{n}}}$. Hence $\pi^{(n)}$ is a counterexample to the semi-positivity theorem.

Corollary 2.7. Let $F$ be a number field and suppose a semi-stable fibration $\pi: X \rightarrow C$ is defined over $F$. There is an integral model of $\pi, \pi_{A}: X_{A} \rightarrow C_{A}$ defined over $\operatorname{Spec} A$, an affine open set of $\operatorname{Spec} \mathcal{O}_{F}$. Let $\pi_{v}: X_{v} \rightarrow C_{v}$ be the reduction of $\pi_{A}$ at a place $v \in \operatorname{Spec} A$. If the genus of $C$ is 0 or 1 , then the generic $p$-rank of $\pi_{v}$ is not 0 for all but finitely many places $v$.

Proof. Since the harder-Narasimhan filtration of $R^{1} \pi_{A *} \mathcal{O}_{X_{A}}$ on the generic fiber of $\mathbb{C}_{A} \rightarrow \operatorname{Spec} A$ extends to a non-empty open set of $\operatorname{Spec} A, R^{1} \pi_{v *} \mathcal{O}_{X_{v}}$ has no positive Harder-Narasimhan slope for almost all $v \in \operatorname{Spec} A$. Therefore the generic $p$-rank of $\pi_{v}$ is not 0 by Theorem 1 .

Let $\mathcal{M}_{g, \mathcal{O}_{F}}$ be the moduli space of proper smooth curves over $\operatorname{Spec} \mathcal{O}_{F} . \mathcal{M}_{g, F}$, the moduli space over $F$, is the generic fiber of $\mathcal{M}_{g, \mathcal{O}_{F}} \rightarrow \operatorname{Spec} \mathcal{O}_{F}$. When $P$ is a geometrically irreducible point of $\mathcal{M}_{g, F}$, the closure of $P$ in $\mathcal{M}_{g, \mathcal{O}_{F}}$ has a geometrically irreducible reduction at almost all places $v$. Let us denote the generic point of the reduction at $v$ by $P_{v} . P_{v}$ is contained in a $p$-rank strata in $\mathcal{O}_{g, \bar{k}}$, the moduli space over the residue field at $v$. We may ask the distribution of the $p$-ranks of $P_{v}$. Serre's ordinary reduction conjecture is a problem for closed points in the moduli space. In the language of the moduli space, Corollary 2.7 can be stated as follow.

Corollary 2.8. Suppose that $\pi: X \rightarrow C$ is a non-isotrivial semi-stable fibration of base genus 0 or 1 defined over a number field $F$, and that $U \subseteq C$ is the smooth locus of $\pi . \pi$ defines a non-constant morphism $f: U \rightarrow \mathcal{M}_{g, F}$. Let $P$ be the image of the generic point of $U$ under $f$. Then the reduction $P_{v}$ is not contained in the $p$-rank 0 strata for almost all $v$.

Remark 2.9. If the fiber genus of $\pi: X \rightarrow C$ is 2, Corollary 2.7 holds for arbitrary base $C$. Ekedahl showed that if $\pi: X \rightarrow C$ is a generically super-singular semi-stable fibration of fiber genus 2, then there exist a finite étale cover $f: D \rightarrow C$ and a morphism $g: D \rightarrow \mathbb{P}^{1}$ such that $\pi_{f}: X \times_{C} D \rightarrow D$ is isomorphic to $\pi_{M, g}: X_{M} \times_{\mathbb{P}^{1}} D \rightarrow$ $D$ where $\pi_{M}: X_{M} \rightarrow \mathbb{P}^{1}$ is Moret-Bailly's fibration.[2],p.173 Since a pullback by a finite separable morphism of curves preserves the semi-stability of vector bundles, $R^{1} \pi_{*} \mathcal{O}_{X}$ has a positive Harder-Narasimhan slope. Considering the construction of a semi-stable fibration from a 1-dimensional point in the moduli space(Remark 2.6) and the Grothendieck specialization theorem, Corollary 2.8 holds for an arbitrary non-closed point in $\mathcal{M}_{2, F}$. 
It is natural to expect that Corollary 2.8 holds for an arbitrary non closed point in $\mathcal{M}_{g, F}$ for any $g$, or equivalently that Corollary 2.7 holds for arbitrary base curve $C$. If the result of Proposition 2.12 in [6] is valid over a filed of characteristic 0, i.e. the slope 0 part of $R^{1} \pi_{*} \mathcal{O}_{X}$ is potentially trivial, this expectation is valid. Indeed, in the situation of Corollary 2.7 without the assumption of the base genus, if the slope 0 part, $\left(R^{1} \pi_{*} \mathcal{O}_{X}\right)_{0}$ is potentially trivial, $\left(R^{1} \pi_{v} \mathcal{O}_{X_{v}}\right)_{0}$ is strongly semi-stable for almost all $v \in \operatorname{Spec} A$. On the other hand, by [8],p.660, the negative slope part, $F_{v}^{n *}\left(R^{1} \pi_{*} \mathcal{O}_{X_{v}}\right)_{-}$ has only negative Harder-Narasimhan slopes for any $n$ if the residue characteristic of $v$ is sufficiently large. Therefore the claim follows.

Question 2.10. For an arbitrary geometrically irreducible non-closed point $P$ in $\mathcal{M}_{g, F}$, is the $p$-rank of the reduction $P_{v}$ nonzero for almost all $v \in \operatorname{Spec} \mathcal{O}_{F}$ ?

\section{References}

[1] Deschamps,M. Réduction Semi-Stable, Asterisque 86,1981,1-34.

[2] Ekedahl,T. On Supersingular Curves and Abelian Varieties, Math.Scand,60,1987,151-178.

[3] Faber,C. and Van Der Geer,G. Complete subvarieties of moduli spaces and the Prym map,J.Reine Angew. Math. 573,2004,117-137.

[4] Fujita,T. On Kagler fiber spaces over curves,J.Math.Soc.Japan,30,no.4,1978,779794.

[5] Illusie,L. Ordinarite des intersections completes generales, The Grothendieck Festschrift vol.II, Progress in Mathematics 87,1990,375-405

[6] Jang,J. Generic ordinarity for semi-stable fibrations, preprint,2008.

[7] Moret-Bailly,L. Familles de Courbes et de Varietes Abeliennes sur $\mathbb{P}^{1}$, Asterisque 86,1981,125-140.

[8] Shepherd-Barron,N. Semi-stability and Reduction mod p, Topology 37,1998,659664.

[9] Szpiro,L. Sur Le Theoreme de rigidite de Parsin et Arakelov, Asterisque 64,1979,169-202

[10] Xiao,G. Surfaces fibrees en courbes de genre deux, Lecture Notes in Mathematics 1137,1980 . 